\title{
NONLINEAR SCHRÖDINGER EQUATION WITH LANDAU DAMPING ON A HALF-LINE
}

\section{LILIANA ESQUIVEL}

Abstract. We consider the initial-boundary value problem for the modified Schrödinger equation, posed on positive half-line $x>0$ :

$$
\left\{\begin{array}{c}
u_{t}+\mathbb{K} u+i|u|^{2} u=0, t \geqslant 0, x \geqslant 0 ; \\
u(x, 0)=u_{0}(x), x>0 \\
u(0, t)=h(t), t>0 .
\end{array}\right.
$$

where the operator $\mathbb{K}$ is defined as

$$
\mathbb{K}(u)=\alpha u_{x x}+\lambda\left|\partial_{x}\right|^{\gamma} u
$$

with $\alpha \in \mathbb{C}, \lambda>0$ and $\left|\partial_{x}\right|^{\gamma}$ is the module-fractional derivative operator defined by

$$
\left|\partial_{x}\right|^{\gamma} u=R^{\gamma} \partial_{x} u
$$

Here $R^{\gamma}$ is the modified Riesz Potential

$$
R^{\gamma} u=\frac{1}{2 \sqrt{\pi} \sin \left(\frac{\pi}{4}\right)} \int_{0}^{\infty} \frac{\operatorname{sign}(x-y)}{\sqrt{|x-y|}} u(y) d y .
$$

We study the local and global existence in time of solutions to the initial-boundary value problem.

Mathematics subject classification (2010): 35Q55, 35B40.

Keywords and phrases: fractional Schrödinger equation, boundary value, Landau damping.

\section{REFERENCES}

[1] M.P. ÁrcigA, Asymptotics for Nonlinear Evolution Equation with Module-Fractional Derivative on a Half-Line, Boundary Value Problem, vol. 2011, Article ID 946143, 29 pages, 2011.

[2] F. BeniteZ, E. KaIKINA, A Neumann problem for the KdV equation with Landau damping on a half-line, Nonlinear Analysis, 74 (2011), 4682-4697.

[3] T. Cazenave, Semilinear Schrödinger Equations. American Mathematical Soc., 2003.

[4] A. Degasperis, S. V. Manakov, P.M. SAntini, On the initial-boundary value problems for soliton equations, Journal of Experimental and Theoretical Physics Letters 2001, Volume 74, Issue 10, pp $481-485$.

[5] A.S. FoKAS, Integrable nonlinear evolution equations on the half-line, Commun. Math. Phys., 230 (2002), 1-39.

[6] A.S. FoKAS, A.R. Its, L-Y Sung. The nonlinear Schrödinger equation on the half-line, Nonlinearity 18, (4) (2005), 1771-1822.

[7] F.D. GakHov, Boundary Value Problems, Dover Publications, INC. New York, 1966.

[8] X. Guo, M. XU, Some physical applications of fractional Schrödinger equation, Journal of Mathematical Physics, 47 (2006), 082104.

[9] B. Guo, Z. Huo, Global Well-Posedness for the Fractional Nonlinear Schrödinger Equation, Communications in Partial Differential Equations, (2010), 247-255. 
[10] A.D. IOnesCU, F. PUS ATERI, Nonlinear fractional Schrödinger equations in one dimension, Journal of Functional Analysis, 266 (2014).

[11] N. HAYASHI, E. KAIKINA, Nonlinear theory of pseudodifferential equations on a half-line. NorthHolland Mathematics Studies, 194. Elsevier Science B. V., Amsterdam, 2004, 319 pp.

[12] N. HAYASHI, P.I. NAUM KIN, Asymptotics of small solutions to nonlinear Schrödinger equations with cubic nonlinearities. Int. J. Pure Appl. Math 3, no. 3 (2002), 255-273.

[13] E. Kaikina, Fractional derivative of Abel type on a Half Line. Transactions of the American Mathematical Society. Vol. 364, No. 10, October 2012, Pages 5149-5172.

[14] E.I. KAIKINA, Asymptotics for inhomogeneous Dirichlet initial-boundary value problem for the nonlinear Schrödinger equation, J. Math. Phys., 54 (2013), no. 11 111504, 15 pp.

[15] N. LAS KIN, Fractional Schrödinger equation

[16] E.K. Lenzi, H.V. Ribeiro, M.A.F. dos Santos, R. Rossato, R. S. Mendes, Time dependent solutions for a fractional Schrödinger equation with delta potentials, Journal of Mathematical Physics, 54, 2013.

[17] S. WANG, M. XU, Generalized fractional Schrödinger equation with space time fractional derivatives, Journal of Mathematical Physics, 48, no. 4, 043502, (2007), 10 pp.

[18] D. WU, Existence and stability of standing waves for nonlinear fractional Schrödinger equations with Hartree type nonlinearity J. Math. Anal. Appl., 411 (2014), 530-542. 